\section{La Relación entre el Nivel de Madurez en la Gestión de Proyectos y los Resultados Caso Joy Global}

\author{
The Relationship Between the Project Management \\ Maturity Level and The Outcomes Case Joy Global
}

\section{RESUMEN}

El principal objetivo de este estudio fue determinar la relación entre el nivel de madurez en la gestión de los proyectos y los resultados del caso Joy Global. Se utilizó como tipo de investigación el tipo descriptivo, y de tipo correlacional, pues tuvo como propósito medir el grado de relación que existe entre las dos variables de estudio. El diseño metodológico es de tipo no experimental. Para el estudio de investigación el nivel de madurez se calcula en función de las 10 áreas de conocimientos del Project Management Institute (PMI) como son la gestión de la integración, alcance, tiempo, costo, calidad, Recursos Humanos (RRHH), comunicación, riesgos, adquisición e interesados y el éxito de un proyecto se calcula teniendo en cuenta las 5 componente de Shenhar y Dvir (2007). Los resultados obtenidos reflejan la relación directa entre la variable nivel de madurez sobre la variable éxito en los proyectos dentro de la empresa Joy Global (Perú).

Palabras claves: Gestión de proyectos; proyecto; modelo de madurez; éxito de proyectos.

\begin{abstract}
The main objective of this study was to determine the relationship between the project management maturity level and the results case Joy Global. The descriptive type and the correlational type were used as the type of research, since its purpose was to measure the degree of relationship that exists between the two study variables. The methodological design is non-experimental. For the research study the maturity level is calculated according to the 10 knowledge areas of the PMI such as integration, scope, time, cost, quality, human resources, communication, risks, acquisition and stakeholders management and the success of a project taking into account the 5 components of (Shenhar \& Dvir, 2007). The results obtained reflect the direct relationship between the maturity level variable on the projects success variable within the company Joy Global (Peru).
\end{abstract}

Keywords: Project management; project; maturity model; project success.

\section{Roger Milián Gonzales}

roger.milian@unmsm.edu.pe

Universidad Nacional Mayor de San Marcos, Facultad de Ciencias Administrativas. Lima, Perú

Presentado: 10/02/2020 - Aceptado: 21/05/2020 - Publicado: 20/10/2020

(C) Los autores. Este artículo es publicado por Gestión en el Tercer Milenio de la Facultad de Ciencias Administrativas de la Universidad Nacional Mayor de San Marcos. Este es un artículo de acceso abierto, distribuido bajo los términos de la licencia Creative Commons Atribucion - No Comercia_Compartir Igual 4.0 Internacional. (http://creativecommons.org/licenses/by-nc-sa/4.0/) que permite el uso no comercial, distribución y reproducción en cualquier medio, siempre que la obra original sea debidamente citada. 


\section{INTRODUCCIÓN}

La investigación tiene como principal objetivo determinar la relación entre el nivel de madurez en la gestión de los proyectos y los resultados que se obtienen dentro de la compañía Joy Global (Perú). Este estudio comenzó con una breve reseña de nuestro contexto económico, el desarrollo de un modelo de madurez en la dirección de proyectos y una metodología de análisis para evaluar los procesos en la dirección de proyectos.

Dentro del contexto económico, el crecimiento en el Perú en los últimos años se debe básicamente a una demanda externa (exportaciones) de materias primas y la ejecución de proyectos con inversión privada y pública en los distintos sectores de la industria peruana. Según el Ministerio de Economía y Finanzas (2019) señala que en el 2019 la inversión privada se fortalecerá como un eje primordial para el desarrollo de la economía, el incremento se debe fundamentalmente a una importante inversión del sector minero y la concatenación que esto origina en los distintos sectores como el de construcción y servicios. De igual forma, la inversión en el sector no minero mantendrá incrementándose debido al restablecimiento de la inversión referente a infraestructura y expectativas convenientes para la inversión en los demás sectores como el retail e inmobiliario. Añadiendo el fomento de la inversión privada, la inversión pública aumentará en $4.5 \%$ en el 2019. Las inversiones tanto pública como privada reforzarán el ciclo inversión-empleo-consumo y posibilitará que el consumo privado llegue a un aumento en la tasa en $3.8 \%$.

Dentro del sector minería se encuentra Joy Global (Perú) que es una empresa de servicios dedicada a la comercialización y reparación de equipos para la minería de superficie y subterránea, incluyendo servicios de reparación de componentes en centros de servicios. Dentro de la unidad de negocios de la compañía se observó que proyectos similares se ejecutan con un enfoque diferente y a la vez no se obtienen el mismo éxito deseado en todos los proyectos y en consecuencia se producen desviaciones en el alcance, tiempo y costos, esto se debe a que los proyectos no se encuentran centralizados dentro de la compañía, ya que no cuenta con una Oficina de
Gestión de Proyectos (PMO) que se encargue de gobernar y estandarizar los proyectos.

Así como Joy Global varias empresas en los distintos sectores están proyectando sus operaciones y procesos para planificar, gestionar y culminar sus proyectos con mayor probabilidad de éxito. Un argumento principal para esta proyección es que actualmente los gerentes de proyectos están en constante presión para que puedan integrar todos los procesos que se encuentran dentro de un proyecto, así como puedan realizar una adecuada planificación y puedan controlar todos los esfuerzos con la finalidad de mejorar el desempeño dentro de los proyectos y la organización, sin embargo cada vez es más difícil poder justificar la inversión financiera en herramientas que ayuden a la gestión de los proyectos.

Según Ibbs y Kwak (2000) señalan que uno de los motivos de esta complicación es que no existen metodologías en dirección de proyectos que tengan una aceptación global o procesos bien establecidos para medir de manera objetiva las buenas prácticas en la gestión de proyectos en cualquier tipo de empresas o en diferentes industrias. Por ejemplo, la guía de los fundamentos para la dirección de proyectos (Guía PMBOK ®), si bien es una fuente autorizada en brindar directrices e información en la gestión de proyectos y aceptada por muchas industrias, carece de este nivel de detalle. El efecto es que muchas empresas tienen dificultades significativas para poner en marcha o mejorar sus procesos de gestión de proyectos. Esto es particularmente fundamental para los gerentes que tienen que sustentar de manera correcta y minuciosa la financiación de la implementación de procesos y prácticas en la gestión de proyectos en sus organizaciones.

Para abordar la deficiencia debemos entender algunos conceptos relacionados a la gestión de proyectos. El Project Management Institute (2017) define que:

Un proyecto como un esfuerzo temporal que se lleva a cabo para crear un producto, servicio o resultado único. La naturaleza temporal de los proyectos implica que un proyecto tiene un principio y un final definidos. El final se alcanza cuando se logran los objetivos del proyecto, 
cuando se termina el proyecto porque sus objetivos no se cumplirán o no pueden ser cumplidos, o cuando ya no existe la necesidad que dio origen al proyecto. Asimismo, se puede poner fin a un proyecto si el cliente (cliente, patrocinador o líder) desea terminar el proyecto. Que sea temporal no significa necesariamente que la duración del proyecto haya de ser corta. Se refiere a los compromisos del proyecto y a su longevidad. (p. 4)

También, el PMI (2017) tiene la idea que la gestión de los proyectos es la concentración de conocimientos, competencias, mecanismos y métodos a los trabajos del proyecto para satisfacer los requerimientos. Esto se obtiene por medio del desarrollo e integración de los 47 procesos de la gestión de proyectos, estructurados de forma lógica, las cuales están agrupadas en 5 categorías de procesos las cuales son: el inicio del proyecto, la planificación, la realización del proyecto, el seguimiento y control, y el cierre.

Por otra parte, desde la perspectiva de los resultados que debe generar un proyecto, Liu y Walker (1998), sostiene que el éxito de un proyecto nos permite tener un concepto distinto para cada individuo, y esto se debe a que cada persona, dependiendo de su opinión o vivencias, poseen una idea distinta acerca de que si un proyecto será o no exitoso. De hecho, Baker, Murphy y Fisher (1988); Dvir, Raz, y Shenhar (2003) afirman que hay muchos casos donde los proyectos se ejecutan según lo planificado, dentro del tiempo, dentro del presupuesto y logran los objetivos de desempeño planificados, pero resultan ser fallas completas porque no lograron producir beneficios reales para el cliente ni los ingresos y beneficios adecuados para la organización ejecutante. Por otro lado, Shenhar, Dvir, Levy y Maltz (2001) manifiestan que con bastante frecuencia lo que parecía ser un proyecto problemático, con grandes retrasos y excesos, resultó ser un gran éxito de negocios. Para Belassi y Tukel (1996) sostienen que también existen proyectos que se perciben de manera exitosa por un gerente e integrantes del equipo del proyecto, pero puede ser percibidos como un error por parte del cliente. Asimismo, existen proyectos considerados exitosos por el cliente y que pueden ser considerados como un fracaso por la alta gerencia, si el resultado del proyecto no cumple con las especificaciones de la alta gerencia, aunque pueda satisfacer al cliente. Tanto del punto de vista de la organización como la del cliente evalúan el éxito del proyecto de manera diferente y, en consecuencia, cada uno valora los resultados de manera diferente.

Según Shenhar y Dvir (2007) muestran un original modelo con la finalidad de garantizar el éxito de los proyectos ampliando la idea que se tenía de la triple restricción (alcance, tiempo y costo) y tiene en cuenta nuevas pautas para el éxito de un proyecto, las cuales abarcar cinco dimensiones: 1) Eficiencia del proyecto: comprender las metas referente al tiempo y presupuesto, 2) Impacto en equipo: satisfacción, retención, y crecimiento personal, 3) Impacto en el cliente: saber las necesidades y obtener la satisfacción del cliente y fidelidad. 4) Resultados del negocio: retorno sobre la inversión, participación de mercado y crecimiento. y 5) Preparación para el futuro: nuevas tecnologías, nuevos mercados, y nuevas capacidades.

Por otro lado, en el contexto de madurez en la gestión de proyectos, Cooke-Davies y Arzymanow (2002) sostienen que la idea de madurez de proceso tuvo sus inicios en la corriente de la Gestión de la Calidad Total, en el cual el desarrollo de instrumentos de seguimiento de los procesos probó que el aumento en la madurez de un proceso lleva, por una parte, a minimizar su variación y, por otra parte, a la mejora continua del desempeño promedio.

El PMI (2013) define "madurez de la dirección de proyectos de una organización como el nivel de capacidad de una organización para producir los resultados estratégicos deseados de un modo predecible, controlable y confiable" (p. 552).

Kwak y Ibbs (2000) explican conceptualmente que la mayoría de los modelos se basan en el Modelo de Madurez de Capacidades del SEI (Instituto de Ingeniería de Software). Varios de los modelos se han descrito en la literatura de gestión de proyectos. Todos ellos definen la madurez de la gestión de proyectos como el nivel de sofisticación de las buenas prácticas y procesos de la dirección de proyectos actuales de una organización. 
Para Crawford (2015) quien desarrolló el Project Management Maturity Model (PMMM), el cual es una herramienta utilizada para medir la madurez en gestión de proyectos de una organización explica que el modelo combina las 10 áreas de conocimiento de la Guía de los Fundamentos para la Gestión de Proyectos (PMBOK) y el Modelo de Madurez de Capacidades (CMM) del Instituto de Ingeniería de Software (SEI). Esta herramienta brinda soluciones de gestión de proyectos y ayuda a los líderes de la Oficina de Gestión de Proyectos (PMO), proyectos y negocios a aplicar prácticas de procesos de gestión de portafolio y proyectos que impulsan el rendimiento y la eficiencia operativa del PMBOK y Modelo de Madurez de capacidades (CMM). El modelo tiene 5 distintos niveles de madurez y analiza su implementación en una organización a través de las 10 áreas de administración de proyectos.

Los 5 niveles del PMMM establecidos por Crawford (2015) análogos al modelo del SEI son: Nivel 1: Proceso Inicial (Initial Process), Nivel 2: Procesos y estándares estructurados, Nivel 3: Procesos institucionalizados y estándares organizacionales (Organizational Standards and Institutionalized Process), Nivel 4: Procesos Gerenciados (Managed Process) y el Nivel 5: Optimización de Procesos (Optimizing Process).

Por lo tanto, es fundamental poder desarrollar y proporcionar a los gerentes un mecanismo para medir los procesos en la gestión de proyectos y el valor de incorporar esos procesos en sus prácticas dentro su organización, por ello se desea determinar el nivel de madurez en la que se encuentra la compañía y cómo se relaciona en la obtención de los resultados en términos de éxito en los proyectos, así como poder establecer una propuesta en la gestión de los procesos y contribuir a la creación de una ventaja competitiva. Por ello se plantea probar las siguientes hipótesis H0: La madurez en gestión de proyectos no se relaciona con el éxito de los proyectos en la empresa Joy Global (Perú) y H1: La madurez en gestión de proyectos se relaciona con el éxito de los proyectos en la empresa Joy Global (Perú). Este análisis ayudaría a los gerentes a evaluar qué prácticas serían las más adecuadas para la organización. Además, ayudaría a las empresas a impulsar las mejores prácticas en la gestión de proyectos.

\section{MÉTODOS}

La dirección de proyectos y la madurez en la dirección de proyectos es una realidad compleja. En ese sentido, se utilizó como tipo de investigación el tipo descriptivo, pues se centra en medir y evaluar dimensiones y elementos asociados a la madurez en la gestión de proyectos y de tipo correlacional, pues tuvo como propósito medir el grado de relación que existe entre las dos variables de estudio. El diseño metodológico es de tipo no experimental, dado que no se manipularán las variables en un laboratorio.

La población de estudio fue la compañía Joy Global (Perú) SAC, de las cuales la muestra fue de 22 colaboradores entre gerentes y líderes de área. Para el estudio se realizó un muestreo no probabilístico intencional, dado que la elección de los elementos no depende de la probabilidad, sino de los objetivos propuestos relacionados con las características de la presente investigación. Se realizó una encuesta a cada uno de los empleados dentro de la organización seleccionados. Para el análisis e interpretación de la información y de los resultados de las pruebas se utilizó estadísticas de asociación entre variables, como el índice Chi-cuadrado de Pearson y coeficientes de correlación, con ayuda de Excel y el SPSS. El instrumento de investigación de esta encuesta consiste en un cuestionario basados en el modelo de Crawford (2015) para obtener el nivel de madurez de la organización y el modelo de Shenhar y Dvir (2007) para medir el éxito de los proyectos.

\section{RESULTADOS}

La investigación analiza el nivel de madurez de la empresa Joy Global y su relación con los resultados dentro de la organización y ayuda a entender la situación actual de la compañía y establecer planes de acción que ayudan a contribuir a mejorar los procesos de la empresa.

El trabajo se ha basado en encuestas que han sido respondidas por aquellas personas que se desempeñan como gerentes y líderes de proyectos que desarrollan funciones asociadas a proyectos y que disponen de conocimientos sobre metodologías relacionados a la gestión de proyectos dentro de la empresa Joy Global (Perú). 
A continuación, se mostrarán los resultados en relación con la empresa para que nos ayude a entender su situación actual, posteriormente se mostrará los resultados del nivel de madurez que tuvo la empresa y su relación con los resultados dentro de la organización en términos de éxito en los proyectos.

La Figura 1, presenta resultados del rol de la persona que desempeña dentro de la empresa, El 46\% de los encuestados desempeñan el rol de gerente y jefes, le siguieron los supervisores con un $27 \%$. El $27 \%$ restante estuvo compuesto por especialistas y personal administrativo.

La Figura 2, presenta resultados de los costos promedios en dólares de los proyectos gestionados, el $50 \%$ de los encuestados indicó que el costo promedio de proyectos gestionados asciende a más de 300,000 dólares, el $27 \%$ indicó que el costo promedio está entre los 100,000 y 300,000 dólares. El 23\% restante es menor a 100,000 dólares.

La Figura 3, muestra resultados si la empresa cuenta con una metodología en la dirección de proyectos, el $82 \%$ de los encuestados manifestó que no existe una metodología en gestión de proyectos documentada.

Referente al análisis del nivel de madurez de las diez áreas de conocimiento de la gestión de proyectos comprendió la gestión de la integración, alcance, tiempo, costo, calidad, recursos

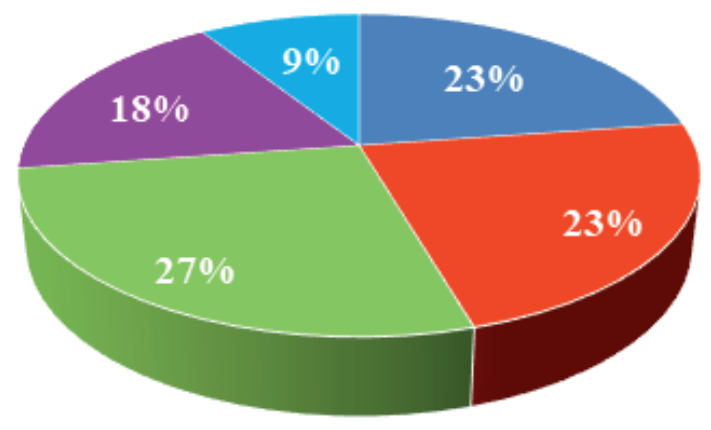

- Gerente - Jefatuta "Supervición - Especialista " Administrativo

Figura 1. Rol que desempeñan dentro de la compañía. Elaboración propia.

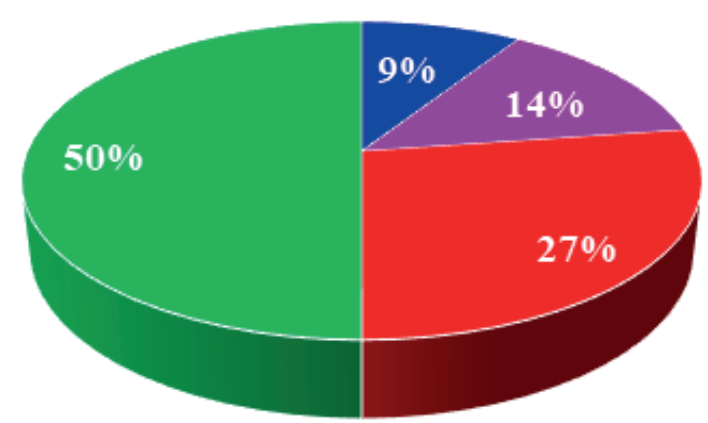

- Hasta 10,000

- Entre 50,000 y 100,000 - Mas de 300,000
Entre 10,000 y 50,000

- Entre 100,000 y 300,000

Figura 2. Costo promedio de cada proyecto gestionado. Elaboración propia. 


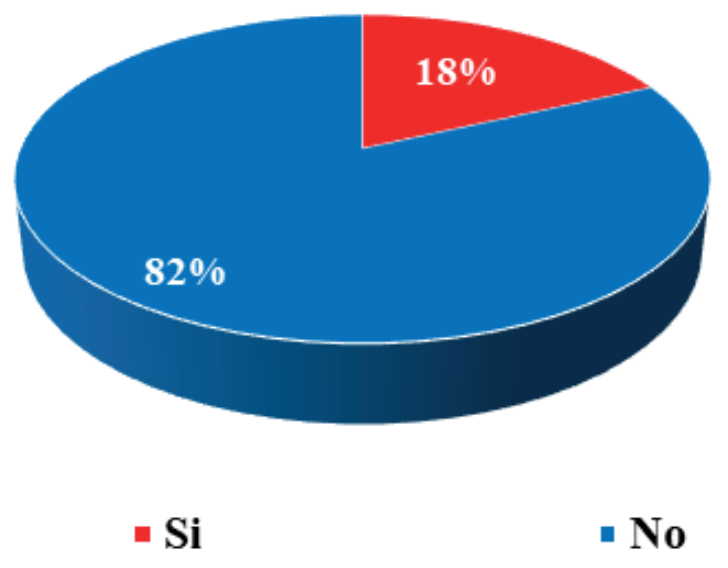

Figura 3. Metodología de proyectos.

Elaboración propia.

humanos, comunicaciones, riesgos, adquisiciones y la gestión de los interesados.

Ahora para conocer el nivel o grado de madurez en gestión de proyectos y por temas didácticos y sea más comprensible para el encuestado se personalizó los niveles de madurez de: Nivel 1 = Procesos Iniciales, Nivel $2=$ Procesos y estándares estructurados, Nivel $3=$ Procesos institucionalizados y estándares organizacionales, Nivel $4=$ Procesos Gerenciados y Nivel $5=$ Optimización de procesos a "Nivel $1=$ Esporádico, Nivel 2 =Limitado, Nivel 3 = Implementado, Nivel $4=$ Controlado $5=$ Optimizado", Lossio, Martínez, y Morris (2016) (p. 29).

La Tabla 1, muestra los resultados del nivel de madurez en la gestión de proyectos dentro de la compañía por cada área de conocimiento y muestra que se encuentra en un nivel 1 (esporádico) y 2 (limitado), es decir utilizan una metodología de proyectos de manera ocasional o limitada dentro de la organización.

A continuación, para el análisis de la relación del nivel de madurez con el éxito en los proyectos se realizó tomando en cuenta el modelo de éxito de un proyecto sugerido por Shenhar y Dvir (2007) basado en 5 dimensiones 1) eficiencia del proyecto, 2) impacto en equipo, 3) impacto en el cliente, 4) resultados del negocio y 5) preparación para el futuro.
La Tabla 2, muestra resultados de la relación que tiene la madurez de cómo se gestionan los proyectos con el éxito de los proyectos dentro de la empresa, y teniendo en cuenta las $5 \mathrm{di}$ mensiones de Shenhar y Dvir (2007), el 77\% de los encuestados indicó que está totalmente de acuerdo que el nivel madurez en gestión de proyectos se relaciona con el éxito de los proyectos, el 23\% de los encuestados indicó que estaba de acuerdo que el nivel de madurez impacta a que la organización pueda conseguir los resultados de éxito en sus proyectos.

La investigación de campo fue delimitada a la empresa Joy Global (Perú), periodo 2017-2018. La encuesta realizada a la compañía nos ha permitido conocer el nivel de madurez en gestión de proyectos que posee actualmente, asimismo su relación con el éxito de los proyectos.

La Tabla 3, muestra resultados de la hipótesis H0: La madurez en gestión de proyectos no se relaciona con el éxito de los proyectos en la empresa Joy Global (Perú), período 20172018 y H1: La madurez en gestión de proyectos se relaciona con éxito de los proyectos en la empresa Joy Global (Perú), período 2017-2018, como el valor de significancia (valor calculado) $0.030<0.05$ rechazamos la hipótesis nula y aceptamos la hipótesis alternativa, es decir el nivel de madurez en la gestión de proyectos de la compañía se relaciona significativamente con el éxito de los proyectos a un nivel de 95\% de confiabilidad 
Tabla 1.

Nivel de Madurez en Gestión de proyectos

\begin{tabular}{|c|c|c|c|c|c|}
\hline Área de Conocimiento Gestión de Proyecto & Nivel 1 & Nivel 2 & Nivel 3 & Nivel 4 & Nivel 5 \\
\hline Integración & $18 \%$ & $77 \%$ & $5 \%$ & $0 \%$ & $0 \%$ \\
\hline Alcance & $5 \%$ & $64 \%$ & $32 \%$ & $0 \%$ & $0 \%$ \\
\hline Tiempo & $9 \%$ & $68 \%$ & $23 \%$ & $0 \%$ & $0 \%$ \\
\hline Costo & $5 \%$ & $86 \%$ & $9 \%$ & $0 \%$ & $0 \%$ \\
\hline Calidad & $27 \%$ & $68 \%$ & $5 \%$ & $0 \%$ & $0 \%$ \\
\hline Recursos Humanos & $14 \%$ & $82 \%$ & $5 \%$ & $0 \%$ & $0 \%$ \\
\hline Comunicaciones & $36 \%$ & $59 \%$ & $5 \%$ & $0 \%$ & $0 \%$ \\
\hline Riesgos & $41 \%$ & $59 \%$ & $0 \%$ & $0 \%$ & $0 \%$ \\
\hline Adquisiciones & $23 \%$ & $64 \%$ & $14 \%$ & $0 \%$ & $0 \%$ \\
\hline Interesados & $9 \%$ & $86 \%$ & $5 \%$ & $0 \%$ & $0 \%$ \\
\hline
\end{tabular}

Fuente. Elaboración propia

Tabla 2.

Relación de la Madurez con los Resultados del Proyecto

\begin{tabular}{lccccc}
$\begin{array}{l}\text { Dimensiones de éxitos de un } \\
\text { Proyecto }\end{array}$ & $\begin{array}{c}\text { Totalmente en } \\
\text { desacuerdo }\end{array}$ & En desacuerdo & Neutral & $\begin{array}{c}\text { De acuerdo } \\
\text { Totalmente de } \\
\text { acuerdo }\end{array}$ & $\begin{array}{c}\text { acu } \\
\text { Eficiencia del proyecto }\end{array}$ \\
Satisfacción del equipo & $0.0 \%$ & $0.0 \%$ & $4.5 \%$ & $27.3 \%$ & $68.2 \%$ \\
Impacto en el cliente & $0.0 \%$ & $0.0 \%$ & $0.0 \%$ & $36.4 \%$ & $77.3 \%$ \\
Resultado del negocio & $0.0 \%$ & $0.0 \%$ & $0.0 \%$ & $22.7 \%$ & $72.7 \%$ \\
Preparación para el futuro & $0.0 \%$ & $0.0 \%$ & $0.0 \%$ & $27.3 \%$ & $59.1 \%$ \\
\hline
\end{tabular}

Fuente. Elaboración propia

Tabla 3.

Nivel de Madurez en Gestión de proyectos*Relación con el éxito de los proyectos

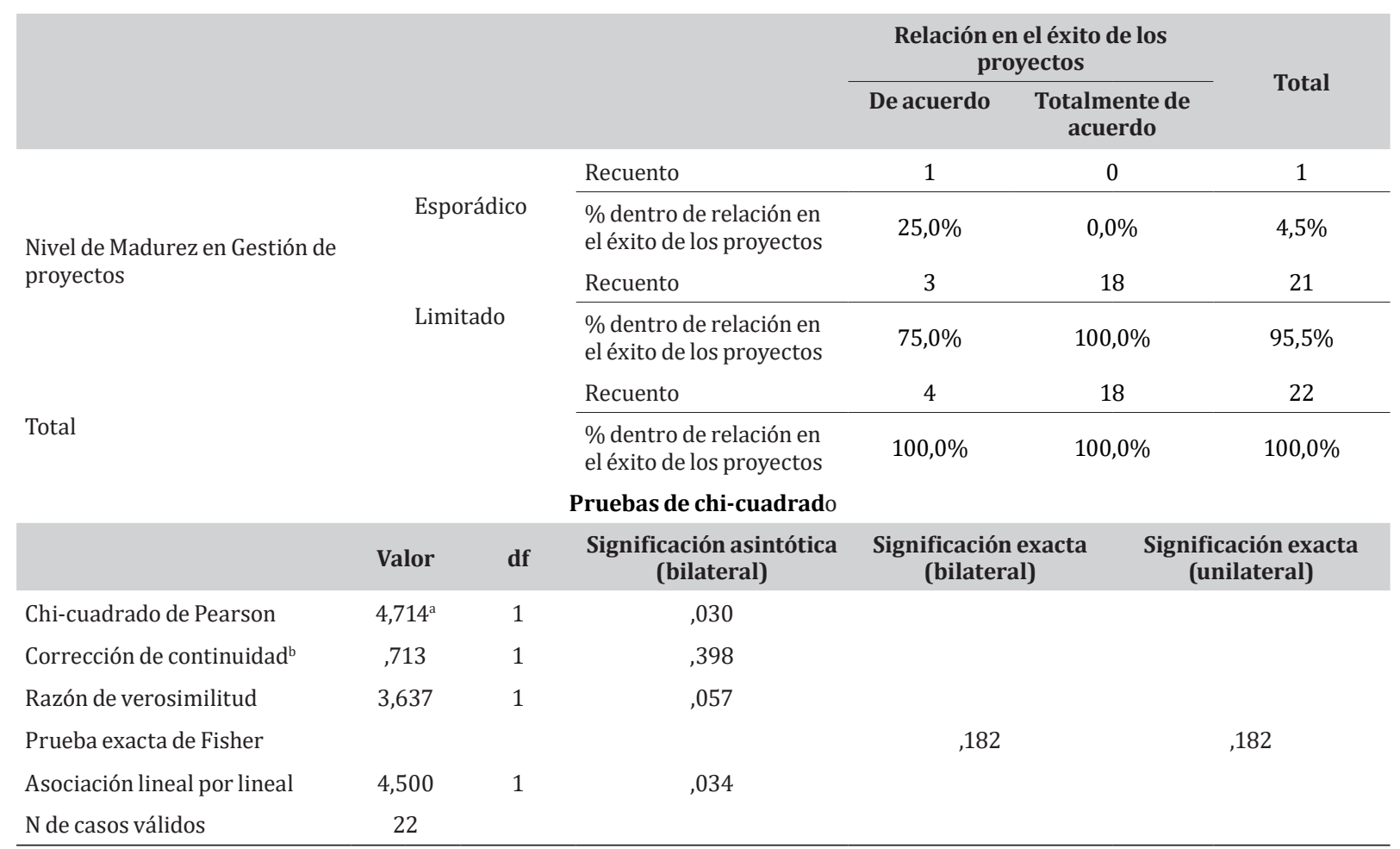

a. 3 casillas $(75,0 \%)$ han esperado un recuento menor que 5 . El recuento mínimo esperado es ,18.

b. Sólo se ha calculado para una tabla $2 \times 2$

Fuente. Elaboración propia con el uso de SPSS 


\section{DISCUSIÓN}

Este estudio muestra las fortalezas y debilidades de las prácticas en la gestión de proyectos entre las áreas de conocimientos dentro de la compañía, además determina las áreas con problemas que dificultan su uso para un gerente dentro del proceso de la gestión de proyectos. Igualmente, la investigación muestra que existe una relación entre el nivel de madurez y los resultados de los proyectos dentro de la empresa Joy Global (Perú). Esto se debe a que si una empresa cuenta con un nivel de madurez bajo o limitado es probable que no se cuente con el éxito deseado en la ejecución de los proyectos. Como Lossio, Martínez, y Morris (2016) mencionan que el nivel de madurez en cómo se administran los proyectos en las empresas peruanas se encuentra en un nivel de madurez limitado y mencionan que es importante determinar y archivar la metodología en gestión de proyectos. A pesar de que un nivel de madurez moderado o optimizado aumenta la probabilidad de tener proyectos exitosos los resultados muestran que la administración de los proyectos en empresas peruanas se encuentra en un nivel limitado. El estudio de investigación realizado es replicable a otros sectores de la industria, ya que conocer el nivel de madurez de la empresa es la etapa inicial para saber dónde se encuentra una compañía y pueda tomar las medidas necesarias para el cumplimiento de sus objetivos estratégicos.

\section{CONCLUSIONES}

La metodología para evaluar el nivel de madurez en gestión de proyectos proporcionó un medio para determinar y medir los diferentes niveles de madurez de las 10 área de conocimientos en la gestión de proyectos bajo un enfoque cuantificado. Adicionalmente según los resultados del presente estudio de investigación, se puede concluir que la gestión de proyectos en la compañía se ubica en un nivel 2 (limitado) y se relaciona significativamente con los resultados en términos de éxito de los proyectos dentro de la compañía. Además la compañía podrá comparar sus procesos empleando técnicas objetivas como las contenidas en el cuestionario, esto dará un punto de referencia sostenible de donde partir y realizar un mejoramiento continuo en los procesos, conjuntamente se recomienda implementar una PMO que pueda proveer metodologías en gestión de proyectos y pueda brindar apoyo para gestionar los proyectos, designar al gestor de proyecto y responsabilizarse de los éxitos y fracasos de los proyectos realizados, y la PMO sea una área de servicios.

\section{REFERENCIAS BIBLIOGRÁFICAS}

Baker, B., Murphy, D. y Fisher, D. (1988). Factors affecting project success. In: Cleland. D.J. King. W.D.. editors. Project Management Handbook. New York: Van Nostrand: 902-919.

Belassi, W. y Tukel, O. (1996). A new framework for determining critical success/failure factors in projects. International Journal of Project Management, 14(3): 141-151.

Cooke-Davies, T. y Arzymanow, A. (2002). The maturity of project management in different industries: International Journal of Project Management.

Crawford, J. (2007). Project Management Maturity Model (Second Edition ed.). Boca Raton: PM Solutions.

Crawford, J. (2015). Project Management Maturity Model (Third Edition ed.). Boca Raton: PM Solutions.

Dvir, D., Raz, T., y Shenhar, A. (2003). An empirical analysis of the relationship between project planning and project success. International Journal of Project Management, 21: 89-95.

Freeman, M., y Beale, P. (1992). Measuring Project Success. Project Management Journal, 23111: 8-17.

Ibss, C. y Kwat, Y. (2000). Assessing project management maturity. Project Management Journal, 31(1), 32-43.

Kwak, Y. y Ibbs, C. (2000). Calculating Project Management's Return on Investment. Project Management Journal, 31(21: 38-47.

Liu, A. y Walker, A. (1998). Evaluation of project outcomes. Construction Management and Economics (16(2)), 209-219.

Lossio, F., Martínez, A. y Morris, E. (2016). La Gestión de Proyectos en el Perú - Análisis de Madurez 2015-2016. Lima: Ediciones Esan.

Ministerio de Economía y Finanzas. (2019). Informe de actualización de proyecciones macroeconómicas 2019-2022. Obtenido de https://www.mef. gob.pe/contenidos/pol_econ/marco_macro/ IAPM_2019_2022.pdf 
pmsolutions. (2012). What is the Project Management Maturity Model (PMMM)? Obtenido de http://www.pmsolutions.com/resources/ view/what-is-the-project-management-maturity-model/

Project Management Institute. (2013). Organizational Project Management Maturity Model (OPM3). Newtown Square, Pennsylvania: Project management Institute, Inc.

Project Management Institute. (2017). A Guide to the project management bdby of knowledge (PMBOK guide). Pennsylvania - EEUU: Project Management Institute, Inc.

Roger, D. y Eder, A. (2020). proyectos. institurte.

Shenhar, A., Dvir, D., Levy, O. y Maltz, A. (2001). Project Success: A Multidimensional Strategic Concept. Long Rang Planning, 34: 699-725.
Shenhar, A. y Dvir, D. (2007). Reinventing project management: The diamond approach to successful growth and innovation. Boston, MA: Harvard Business Press.

Shenhar, A., Levy, O., \& Dvir, D. (1997). Mapping the dimensions of project success. Project Management Journal (28 (2)), 5-13.

Slevin, D. y Pinto, J. (1988). Project success: Definitions and measurement techniques. Project Management Journal.

Solarte-Pazos, L. y Sánchez-Arias, L. (2013). Gerencia de proyectos y estrategia organizacional: el modelo de madurez en Gestión de Proyectos CP3M(C V5.0*. Revista Innovar Journal. 
